\title{
$s$-wave approximation for asymmetry in nonmesonic decay of finite hypernuclei
}

\author{
Cesar Barbero, ${ }^{1,4}$ Alfredo P. Galeão, ${ }^{2}$ and Francisco Krmpoticic $3,4,5$ \\ ${ }^{1}$ Facultad de Ciencias Exactas, Departamento de Física, Universidad Nacional de La Plata, 1900 La Plata, Argentina \\ ${ }^{2}$ Instituto de Física Teórica, Universidade Estadual Paulista, Rua Pamplona 145, 01405-900 São Paulo, SP, Brazil \\ ${ }^{3}$ Departamento de Física Matemática, Instituto de Física da Universidade de São Paulo, Caixa Postal 66318, 05315-970 São Paulo, SP, Brazil \\ ${ }^{4}$ Instituto de Física La Plata, CONICET, 1900 La Plata, Argentina \\ ${ }^{5}$ Facultad de Ciencias Astronómicas y Geofísicas, Universidad Nacional de La Plata, 1900 La Plata, Argentina
}

(Received 22 May 2007; revised manuscript received 21 August 2007; published 28 November 2007)

\begin{abstract}
We establish the bridge between the commonly used Nabetani-Ogaito-Sato-Kishimoto (NOSK) formula for the asymmetry parameter $a_{\Lambda}$ in the $\vec{\Lambda} p \rightarrow n p$ emission of polarized hypernuclei, and the shell-model (SM) formalism for finite hypernuclei. We demonstrate that the $s$-wave approximation leads to a SM formula for $a_{\Lambda}$ that is as simple as the NOSK one and that reproduces the exact results for ${ }_{\Lambda}^{5} \mathrm{He}$ and ${ }_{\Lambda}^{12} \mathrm{C}$ better than initially expected. The simplicity achieved here is indeed remarkable. The new formalism makes the theoretical evaluation of $a_{\Lambda}$ more transparent and explains clearly why the one-meson exchange model is unable to account for the experimental data of ${ }_{\Lambda}^{5} \mathrm{He}$.
\end{abstract}

DOI: 10.1103/PhysRevC.76.054321

PACS number(s): 21.80.+a, 13.75.Ev, 21.60.-n, 27.20.+n

\section{INTRODUCTION}

Despite recent important developments in the $\Lambda N \rightarrow N N$ nonmesonic weak decay (NMWD) [1], its reaction mechanism is not fully understood. Indeed, an open problem concerns the asymmetry parameter $a_{\Lambda}$ in the $\vec{\Lambda} p \rightarrow n p$ emission of the polarized hypernuclei ${ }_{\Lambda}^{5} \overrightarrow{\mathrm{He}}$ and ${ }_{\Lambda}^{12} \overrightarrow{\mathrm{C}}$, which yields information on the interference between parity-conserving (PC) and parity-violating (PV) transitions. The measurements favour $a_{\Lambda}\left({ }_{\Lambda}^{5} \overrightarrow{\mathrm{He}}\right)>0$ and $a_{\Lambda}\left({ }_{\Lambda}^{12} \overrightarrow{\mathrm{C}}\right)<0$ [2], whereas the calculations yield almost the same negative value for both $[1,3]$.

The intrinsic $\Lambda$ asymmetry is usually evaluated from the formula,

$$
a_{\Lambda}=2 \sqrt{3} \frac{\Re\left[a e^{*}-b(c-\sqrt{2} d)^{*} / \sqrt{3}+f(\sqrt{2} c+d)^{*}\right]}{|a|^{2}+|b|^{2}+3\left(|c|^{2}+|d|^{2}+|e|^{2}+|f|^{2}\right)},
$$

where the two-body $|\Lambda p ; l S J\rangle \rightarrow\left|n p ; l^{\prime} S^{\prime} J\right\rangle$ nonmesonic $p \Lambda \rightarrow p n$ decay amplitudes

$$
\begin{aligned}
& a=\left\langle{ }^{1} \mathrm{~S}_{0}|\hat{V}|^{1} \mathrm{~S}_{0}\right\rangle, \quad b=\left\langle{ }^{3} \mathrm{P}_{0}|\hat{V}|{ }^{1} \mathrm{~S}_{0}\right\rangle, \quad c=\left\langle{ }^{3} \mathrm{~S}_{1}|\hat{V}|^{3} \mathrm{~S}_{1}\right\rangle, \\
& d=\left\langle{ }^{3} \mathrm{D}_{1}|\hat{V}|^{3} \mathrm{~S}_{1}\right\rangle, \quad e=\left\langle{ }^{1} \mathrm{P}_{1}|\hat{V}|^{3} \mathrm{~S}_{1}\right\rangle, \quad f=\left\langle{ }^{3} \mathrm{P}_{1}|\hat{V}|^{3} \mathrm{~S}_{1}\right\rangle
\end{aligned}
$$

are the kinematical correspondents of the inverse reaction $p n \rightarrow p \Lambda$. Equation (1.1) was derived by Nabetani, Ogaito, Sato, and Kishimoto (NOSK) [4] considering only the $s$-wave production for the $p \Lambda$ final states. This $s$-wave approximation (s-WA) can be used for the NMWD straightforwardly only in the context of the Fermi gas model (FGM), where the $\Lambda$-hyperon is embedded in the infinite nuclear matter and is taken to be always in a relative $s$-state with respect to any of the nucleons within the hypernucleus [5]. ${ }^{1}$ There are several differences between the scattering states $p \Lambda$ in the reaction

\footnotetext{
${ }^{1}$ Note that the FGM expression [5] Eq. (88)] for $a_{\Lambda}$ is incomplete because it covers only the last term in the numerator of Eq. (1.1).
}

$p n \rightarrow p \Lambda$ and the shell-model (SM) description of the nuclear bound states $p \Lambda$. In the SM the hyperon stays in the $1 s_{1 / 2}$ orbital and, depending on the hypernucleus, the proton can occupy the orbitals $1 s_{1 / 2}, 1 p_{3 / 2}, 1 p_{1 / 2}, \cdots$ It is true that in the case of $1 s_{1 / 2}$-shell hypernuclei, the initial $p \Lambda$ system can be assumed to be in the relative $s$-wave state and therefore it is sufficient to consider only the six matrix elements (1.2). In fact, following the Block-Dalitz anzatz [6] for the employment of the FGM in finite nuclei, one can use the Eq. (1.1) for ${ }_{\Lambda}^{5} \mathrm{He}$ [7]. But in the case of ${ }_{\Lambda}^{12} \mathrm{C}$ both the $1 s_{1 / 2}$ and $1 p_{3 / 2}$ single-particle states contribute and, in addition to the relative $s$ state, one has to consider the relative $p$ state as well [3,8-10].

Based on the above arguments, in our previous work [3], we have derived a SM expression for $a_{\Lambda}$ that is valid for both ${ }_{\Lambda}^{5} \mathrm{He}$ and ${ }_{\Lambda}^{12} \mathrm{C}$. The NOSK formula [Eq. (1.1)] has been used there only for the sake of numerical comparison in the case of ${ }_{\Lambda}^{5} \mathrm{He}$. Here we go a step further, establishing a bridge between the two formalisms. More specifically, we show that under plausible assumptions, the s-WA can also be introduced in the $\mathrm{SM}$, yielding a NOSK-like formula, which can be used in the finite hypernuclei ${ }_{\Lambda}^{5} \mathrm{He}$ and ${ }_{\Lambda}^{12} \mathrm{C}$.

\section{EXACT EXPRESSION FOR $a_{\wedge}$}

To introduce the notation, we give here a short account of the formalism we have developed for the calculation of $a_{\Lambda}$ in Ref. [3], where more details can be found.

The mixed state of a hypernucleus having vector polarization $\boldsymbol{P}_{V}$ can be represented by the density matrix [[11] Eq. (9.29)]

$$
\rho\left(J_{I}\right)=\frac{1}{2 J_{I}+1}\left(1+\frac{3}{J_{I}+1} \boldsymbol{P}_{V} \cdot \boldsymbol{J}_{I}\right),
$$

where $J_{I}$ is the hypernuclear spin. The angular distribution of primary protons emitted by such a hypernucleus is then given 
by

$$
\begin{aligned}
& \frac{d \Gamma\left[\rho\left(J_{I}\right) \rightarrow \hat{\boldsymbol{p}}_{2} t_{p}\right]}{d \Omega_{p_{2}}} \\
& =\int d \Omega_{p_{1}} \int d F \sum_{s_{1} s_{2} M_{F}} \sum_{M_{I} M_{I}^{\prime}}\left\langle\boldsymbol{p}_{1} s_{1} t_{n} \boldsymbol{p}_{2} s_{2} t_{p} v_{F} J_{F} M_{F}\right| \\
& \quad \times V\left|J_{I} M_{I}\right\rangle\left\langle J_{I} M_{I}\left|\rho\left(J_{I}\right)\right| J_{I} M_{I}^{\prime}\right\rangle \\
& \quad \times\left\langle J_{I} M_{I}^{\prime}\left|V^{\dagger}\right| \boldsymbol{p}_{1} s_{1} t_{n} \boldsymbol{p}_{2} s_{2} t_{p} v_{F} J_{F} M_{F}\right\rangle
\end{aligned}
$$

where $V$ is the nonmesonic transition potential, $\boldsymbol{p}_{1} s_{1} t_{n} \equiv$ $-1 / 2$ and $\boldsymbol{p}_{2} s_{2} t_{p} \equiv+1 / 2$ are the momenta and spin and isospin projections of the emitted neutron and proton, respectively, and $\left|v_{F} J_{F} M_{F}\right\rangle$ are the possible final states of the residual nucleus, where $v_{F}$ specifies the remaining quantum numbers in addition to those related to the nuclear spin. Furthermore, we have introduced the compact notation

$$
\begin{aligned}
& \int d F \ldots \\
& =2 \pi \sum_{\nu_{F} J_{F}} \int \frac{p_{2}^{2} d p_{2}}{(2 \pi)^{3}} \int \frac{p_{1}^{2} d p_{1}}{(2 \pi)^{3}} \\
& \quad \times \delta\left(\frac{p_{1}^{2}}{2 M_{N}}+\frac{p_{2}^{2}}{2 M_{N}}+\frac{\left|\boldsymbol{p}_{1}+\boldsymbol{p}_{2}\right|^{2}}{2 M_{R}}-\Delta_{v_{F} J_{F}}\right) \ldots
\end{aligned}
$$

where the $\delta$ function enforces energy conservation, $M_{R}$ is mass of the residual nucleus, and $\Delta_{v_{F} J_{F}}$ is the liberated energy. form

It is possible to show that the Eq. (2.2) can be put in the

$$
\frac{d \Gamma\left[\rho\left(J_{I}\right) \rightarrow \hat{\boldsymbol{p}}_{2} t_{p}\right]}{d \Omega_{p_{2}}}=\frac{\Gamma_{p}}{4 \pi}\left(1+A_{V} \boldsymbol{P}_{V} \cdot \hat{\boldsymbol{p}}_{2}\right),
$$

where $\Gamma_{p}$ is the full proton-induced decay rate and $A_{V}$ is the vector hypernuclear asymmetry, given by

$$
A_{V}=\frac{3}{J_{I}+1} \frac{\sum_{M_{I}} M_{I} \sigma\left(J_{I} M_{I}\right)}{\sum_{M_{I}} \sigma\left(J_{I} M_{I}\right)} .
$$

The new quantities introduced above are the decay strengths,

$$
\begin{aligned}
\sigma\left(J_{I} M_{I}\right)= & \int d \Omega_{p_{1}} \int d F \sum_{s_{1} s_{2} M_{F}} \mid\left\langle\boldsymbol{p}_{1} s_{1} t_{n} \boldsymbol{p}_{2} s_{2} t_{p} v_{F} J_{F} M_{F}\right| \\
& \times\left. V\left|J_{I} M_{I}\right\rangle_{\text {p.h.f. }}\right|^{2}
\end{aligned}
$$

where the subscript p.h.f. indicates that the transition amplitude must be computed in the proton helicity frame.

To proceed, one must write the transition amplitudes in Eq. (2.6) in terms of the two-body matrix elements for the elementary process $\Lambda p \rightarrow n p$ occurring between the appropriate bound $\Lambda p$ states in the hypernucleus and the allowed free final $n p$ states. To this end it is convenient to work in the total spin $\left(S, M_{S}\right)$ and isospin $\left(T, M_{T}\right)$ basis and to change the representation to relative and total momenta, given, respectively, by $\boldsymbol{p}=\left(\boldsymbol{p}_{2}-\boldsymbol{p}_{1}\right) / 2$ and $\boldsymbol{P}=\boldsymbol{p}_{1}+\boldsymbol{p}_{2}$.
Dropping the $M_{T}=0$ labels, one obtains

$$
\begin{aligned}
& \sigma\left(J_{I} M_{I}\right) \\
& =\int d \Omega_{p_{1}} \int d F \sum_{S M_{S} M_{F}} \mid \sum_{T}(-)^{T}\left\langle\boldsymbol{p} \boldsymbol{P} S M_{S} T v_{F} J_{F} M_{F}\right| \\
& \quad \times\left. V\left|J_{I} M_{I}\right\rangle_{\text {p.h.f. }}\right|^{2}
\end{aligned}
$$

Next, we (i) expand the final state in terms of the relative $(\boldsymbol{l})$ and center-of-mass $(\boldsymbol{L})$ partial waves of the emitted nucleons [[8] (2.5)], (ii) drop the subscript p.h.f. due to the rotational invariance of $V$, and (iii) integrate on the angle $\phi_{p_{1}}$, to obtain

$$
\begin{aligned}
\sigma\left(J_{I} M_{I}\right)= & \frac{(4 \pi)^{5}}{2} \int d \cos \theta_{p_{1}} \int d F \sum_{S M_{S} M_{F}} \\
& \times \mid \sum_{l L \lambda J T}(-)^{T} i^{-l-L}\left[Y_{l}\left(\theta_{p}, \pi\right) \otimes Y_{L}\left(\theta_{P}, 0\right)\right]_{\lambda \mu} \\
& \times\left(\lambda \mu S M_{S} \mid J M_{J}\right)\left(J M_{J} J_{F} M_{F} \mid J_{I} M_{I}\right) \\
& \times\left.\left\langle p l P L \lambda S J T v_{F} J_{F} ; J_{I}|V| J_{I}\right\rangle\right|^{2}
\end{aligned}
$$

where $\lambda=\boldsymbol{l}+\boldsymbol{L}$ and $\boldsymbol{J}=\boldsymbol{\lambda}+\boldsymbol{S}$, and

$$
\begin{aligned}
4 p^{2} & =p_{1}^{2}+p_{2}^{2}-2 p_{1} p_{2} \cos \theta_{p_{1}} \\
P^{2} & =p_{1}^{2}+p_{2}^{2}+2 p_{1} p_{2} \cos \theta_{p_{1}} \\
\cos \theta_{p} & =\frac{p_{2}-p_{1} \cos \theta_{p_{1}}}{2 p} \\
\cos \theta_{P} & =\frac{p_{2}+p_{1} \cos \theta_{p_{1}}}{P}
\end{aligned}
$$

where $\theta_{p_{1}}$ is the angle that $\boldsymbol{p}_{1}$ makes with $\boldsymbol{p}_{2}$.

Afterward, we rewrite the Eq. (2.5) for $A_{V}$ in terms of the decay moments

$$
\begin{aligned}
& \sigma_{0}\left(J_{I}\right)=\sum_{M_{I}} \sigma\left(J_{I} M_{I}\right), \\
& \sigma_{1}\left(J_{I}\right)=\frac{1}{\sqrt{J_{I}\left(J_{I}+1\right)}} \sum_{M_{I}} M_{I} \sigma\left(J_{I} M_{I}\right),
\end{aligned}
$$

as

$$
A_{V}=3 \sqrt{\frac{J_{I}}{J_{I}+1}} \frac{\sigma_{1}\left(J_{I}\right)}{\sigma_{0}\left(J_{I}\right)}
$$

We remark that the summations on $M_{S}, M_{F}$, and $M_{I}$ have been explicitly performed in Ref. [3] [cf. Eqs. (21) and (27) in that reference].

Moreover, we adopt here both (i) the weak-coupling model (WCM), where for the ${ }^{A-1} Z$ core ground state $\left|J_{C}\right\rangle$, the initial state is $\left|J_{I}\right\rangle \equiv\left|\left(\mathrm{j}_{\Lambda} J_{C}\right) J_{I}\right\rangle$ and (ii) the extreme shell model (ESM), where $\left|v_{F} J_{F}\right\rangle \equiv\left|\left(\mathrm{j}_{p}^{-1} J_{C}\right) J_{F}\right\rangle$. $\mathrm{j}_{\Lambda} \equiv n_{\Lambda} l_{\Lambda} j_{\Lambda}$ and $\mathrm{j}_{p} \equiv n_{p} l_{p} j_{p}$ are the single-particle states for the $\Lambda$ and proton, respectively. Under these circumstances, and when the single-proton subshells are completely filled in $\left|J_{C}\right\rangle$, as happens in the case of ${ }^{5} \mathrm{He}$ and ${ }^{12} \mathrm{C}$, 
one gets:

$$
\begin{aligned}
& \left\langle p l P L \lambda S J T v_{F} J_{F} ; J_{I}|V| J_{I}\right\rangle \\
& =(-)^{J_{C}-J_{F}+j_{p}} \hat{J} \hat{J}_{F}\left\{\begin{array}{lll}
J_{C} & J_{I} & j_{\Lambda} \\
J & j_{p} & J_{F}
\end{array}\right\} \mathcal{M}(p l P L \lambda S J T ; \Lambda \mathrm{p})
\end{aligned}
$$

with

$$
\begin{aligned}
& \mathcal{M}(p l P L \lambda S J T ; \Lambda \mathrm{p}) \\
& \quad=\frac{1}{2}\left[1-(-)^{l+S+T}\right](-)^{T+1}\left(p l P L \lambda S J T|V| \mathrm{j}_{\Lambda} \mathrm{j}_{p} J T\right),
\end{aligned}
$$

where the compact notation $\Lambda \equiv \mathrm{j}_{\Lambda}, t_{\Lambda}=-1 / 2, \mathrm{p} \equiv \mathrm{j}_{p}, t_{p}$ has been used, and the isospin coupling $\left|t_{\Lambda} t_{p}\right\rangle=1 / \sqrt{2}(\mid T=$ $\left.\left.1, M_{T}=0\right\rangle-\left|T=0, M_{T}=0\right\rangle\right)$ has been carried out.

Within the above description, the liberated energies are independent of $J_{F}$, i.e. $\Delta_{v_{F} J_{F}} \rightarrow \Delta_{\mathrm{j}_{p}}=M_{\Lambda}-M_{N}+\epsilon_{\mathrm{j}_{\Lambda}}+$ $\epsilon_{\mathrm{j}_{p}}$, where the $\epsilon$ 's are separation energies and $M_{\Lambda}$ is the hyperon mass. Forthwith, we rewrite the integration in Eq. (2.8) as

$$
\int d \cos \theta_{p_{1}} \int d F \ldots=\frac{1}{(2 \pi)^{5}} \sum_{\mathrm{j}_{p}} \int d U_{\mathrm{j}_{p}} \sum_{J_{F}} \ldots,
$$

where

$$
\begin{aligned}
& \int d U_{\mathrm{j}_{p}} \cdots=\int d \cos \theta_{p_{1}} \int p_{2}^{2} d p_{2} \int p_{1}^{2} d p_{1} \\
& \quad \times \delta\left(\frac{p_{1}^{2}}{2 M_{N}}+\frac{p_{2}^{2}}{2 M_{N}}+\frac{\left|\mathbf{p}_{1}+\mathbf{p}_{\mathbf{2}}\right|^{2}}{2 M_{R}}-\Delta_{\mathrm{j}_{p}}\right) \cdots,
\end{aligned}
$$

Putting all this together, and performing the summation on $J_{F}$, we end up with the decay moments $\sigma_{\kappa}\left(J_{I}\right)$, given by Ref. [3, Eq. (34)], that have a purely kinematical dependence on the hypernuclear spin $J_{I}$. This dependence can be eliminated within the WCM by defining [12] the intrinsic asymmetry parameter

$$
a_{\Lambda}=\left\{\begin{array}{lll}
A_{V} & \text { for } & J_{I}=J_{C}+1 / 2 \\
-\frac{J_{I}+1}{J_{I}} A_{V} & \text { for } & J_{I}=J_{C}-1 / 2
\end{array}\right.
$$

which in the formalism explained above takes the form [3]:

$$
a_{\Lambda}=\frac{\omega_{1}}{\omega_{0}}
$$

with the decay moments

$$
\begin{aligned}
& \omega_{\kappa}=(-)^{\kappa} \frac{8}{\sqrt{2 \pi}} \hat{\kappa}^{-1} \sum_{\mathrm{j}_{p}} \int d U_{\mathrm{j}_{p}} Y_{\kappa 0}\left(\theta_{p}, 0\right) \\
& \times \sum_{T T^{\prime}}(-)^{T+T^{\prime}} \sum_{L S} \sum_{l \lambda J} \sum_{l^{\prime} \lambda^{\prime} J^{\prime}} i^{l-l^{\prime}} \\
& \times(-)^{\lambda+\lambda^{\prime}+S+L+j_{p}+\frac{1}{2}} \hat{l} \hat{l}^{\prime} \hat{\lambda} \hat{\lambda}^{\prime} \hat{J}^{2} \hat{J}^{\prime 2}\left(l 0 l^{\prime} 0 \mid \kappa 0\right) \\
& \times\left\{\begin{array}{ccc}
\kappa & 1 / 2 & 1 / 2 \\
j_{p} & J & J^{\prime}
\end{array}\right\}\left\{\begin{array}{ccc}
\kappa & J^{\prime} & J \\
S & \lambda & \lambda^{\prime}
\end{array}\right\}\left\{\begin{array}{lll}
l^{\prime} & l & \kappa \\
\lambda & \lambda^{\prime} & L
\end{array}\right\} \\
& \times \mathcal{M}(p l P L \lambda S J T ; \Lambda p) \mathcal{M}^{*}\left(p l^{\prime} P L \lambda^{\prime} S J^{\prime} T^{\prime} ; \Lambda \mathrm{p}\right) \text {, }
\end{aligned}
$$

where $\hat{J}=\sqrt{2 J+1}$, etc. We note that the moments $\omega_{\kappa}$ do not depend on the hypernuclear spin $J_{I}$, and that $L=0$ for the $1 s_{1 / 2}$ state, and $L=0$ and 1 for the $1 p_{3 / 2}$ state.

To evaluate the matrix elements in Eq. (2.13) one has to carry out the $j j-L S$ recouping and the Moshinsky transformation [13] on the ket $\mid \mathrm{j}_{\Lambda} \mathrm{j}_{p} J T$ ) (see Ref. [8, Eq. (2.14)]) to get

$$
\begin{aligned}
& \left(p l P L \lambda S J T|V| \mathrm{j}_{\Lambda} \mathrm{j}_{p} J T\right) \\
& =\hat{j}_{\Lambda} \hat{j}_{p} \sum_{\lambda^{\prime} S^{\prime} \mathrm{nINL}} \hat{\lambda}^{\prime} \hat{S}^{\prime}\left\{\begin{array}{ccc}
l_{\Lambda} & \frac{1}{2} & j_{\Lambda} \\
l_{p} & \frac{1}{2} & j_{p} \\
\lambda^{\prime} & S^{\prime} & J
\end{array}\right\}(P L \mid \mathrm{NL}) \\
& \quad \times\left(\mathrm{nINL} \lambda^{\prime} \mid n_{\Lambda} l_{\Lambda} n_{p} l_{p} \lambda^{\prime}\right)\left(p, l L \lambda S J ; T|V| \mathrm{nIL} \lambda^{\prime} S^{\prime} J ; T\right),
\end{aligned}
$$

where $\left(\mathrm{n} \cdots \mid n_{\Lambda} \cdots\right)$ are the Moshinsky brackets [13]. Here, I and $L$ stand for the quantum numbers of the relative and center-of-mass orbital angular momenta in the $\Lambda N$ system. Moreover,

$$
(P L \mid \mathrm{NL})=\delta_{L, \mathrm{~L}} \int R^{2} d R j_{L}(P R) \mathcal{R}_{\mathrm{NL}}(R),
$$

is the overlap of the center-of-mass radial wave functions. One is interested here in the $\mathrm{j}_{p}=1 s_{1 / 2}$ state, for which is $\mathrm{I}=\mathrm{L}=0$, and in the $\mathrm{j}_{p}=1 p_{3 / 2}$ state, for which both $\mathrm{I}=0, \mathrm{~L}=1$, and $\mathrm{I}=1, \mathrm{~L}=0$ terms contribute.

\section{APPROXIMATE EXPRESSION FOR $a_{\Lambda}$}

We start this section by neglecting the kinematical and nonlocal effects on the NMWD introduced in Ref. [10], which, as shown there and confirmed in Ref. [3], do not affect the final results by more than 10-20\%. Afterwards we write the Eq. (2.18) in the form:

$$
\omega_{\kappa}=\frac{8}{\sqrt{\pi}} \sum_{\mathrm{j}_{p} \mathrm{~L}} \int d U_{\mathrm{j}_{p}} Y_{\kappa 0}\left(\theta_{p}, 0\right) \mathcal{O}(P ; \mathrm{L}) \mathcal{I}_{\kappa}\left(p ; \mathrm{j}_{p} \mathrm{l}\right),
$$

where $\mathcal{O}(P ; \mathrm{L}) \equiv(P \mathrm{~L} \mid 1 \mathrm{~L})^{2}$ and

$$
\mathcal{O}(P ; 0)=\sqrt{\frac{\pi}{2}} b^{3} e^{-(P b)^{2} / 2} ; \quad \mathcal{O}(P ; 1)=\frac{(b P)^{2}}{3} \mathcal{O}(P ; 0),
$$

with $b$ being the oscillator length [8].

From Eqs. (2.18), (2.13), and (2.19) one sees that the just-introduced quantities $\mathcal{I}_{\kappa}\left(p ; \mathrm{j}_{p} l\right)$ are complicated function of $p, \mathrm{j}_{p}$, and I. They involve several Racah coefficients and many summations on different angular momenta and isospins. However, after performing all the algebra analytically, we have demonstrated that the nuclear amplitudes $\mathcal{I}_{\kappa}\left(p ; \mathrm{j}_{p} \mathrm{I}=0\right)$ do not depend on $\mathrm{j}_{p}$, i.e.

$$
\mathcal{I}_{\kappa}\left(p ; \mathrm{j}_{p}=1 s_{1 / 2}, 0\right)=\mathcal{I}_{\kappa}\left(p ; \mathrm{j}_{p}=1 p_{3 / 2}, 0\right) \equiv \mathcal{I}_{\kappa}(p ; 0) .
$$

However, for $\mathrm{I}=1$ only the $\mathrm{j}_{p}=1 p_{3 / 2}$ state contributes, and one can write

$$
\mathcal{I}_{\kappa}\left(p ; \mathrm{j}_{p}=1 p_{3 / 2}, 1\right) \equiv \mathcal{I}_{\kappa}(p ; 1)
$$


The explicit expressions for the form quantities $\mathcal{I}_{\kappa}(p ; l)$, in the one-meson-exchange model (OMEM), that comprise the $\left(\pi, \eta, K, \rho, \omega, K^{*}\right)$ mesons, are exhibited in Appendix A. The $\kappa=0$ pieces of Eq. (3.1), i.e. the Eqs. (A1) and (A3), have already been derived in a previous work [8], where we have also learned that the matrix elements contained within $\mathcal{I}_{0}(p ; 1)$ represent the higher order contributions (HOC), when compared with those contained within $\mathcal{I}_{0}(p ; 0)$. These HOC are $\cong 2 \%$ for the PC transitions and $\cong 15 \%$ for the PV transitions (see also Ref. [14]). Here we have verified numerically that the HOC contribute to $\omega_{1}$ in similar proportions, and therefore their overall effect on $a_{\Lambda}$ is relatively small. Thus, the $\mathrm{I}=1$ contributions to ${ }_{\Lambda}^{12} \mathrm{C}$, will be omitted from now on, and we end up with

$$
\omega_{\kappa}=\frac{8}{\sqrt{\pi}} \sum_{\mathrm{j}_{p} \mathrm{~L}} \int d U_{\mathrm{j}_{p}} Y_{\kappa 0}\left(\theta_{p}, 0\right) \mathcal{O}(P ; \mathrm{L}) \mathcal{I}_{\kappa}(p ; 0),
$$

which, together with Eq. (2.17) is what we call the s-WA for $a_{\Lambda}$ in finite hypernuclei. Needless to say that Eq. (3.5) is exact for ${ }_{\Lambda}^{5} \mathrm{He}$ and equivalent to Eq. (2.18). Note that the summation on $\mathrm{j}_{p}$ in Eq. (3.5) affects only the range of the integration as indicated in Eq. (2.15).

Next we show that the amplitudes $\mathcal{I}_{0}(p ; \mid=0)$ and $\mathcal{I}_{1}(p ; \mid=$ $0)$ exhibit the same combination of nuclear matrix elements as the numerator and the denominator in Eq. (1.1). That is:

$$
\begin{aligned}
& \mathcal{I}_{0}(p ; 0)=|\mathrm{a}|^{2}+|\mathrm{b}|^{2}+3\left(|\mathrm{c}|^{2}+|\mathrm{d}|^{2}+|\mathrm{e}|^{2}+|\mathrm{f}|^{2}\right), \\
& \mathcal{I}_{1}(p ; 0)=2 \Re\left[a e^{*}-\mathrm{b}(\mathrm{c}-\sqrt{2} \mathrm{~d})^{*} / \sqrt{3}+\mathrm{f}(\sqrt{2} \mathrm{c}+\mathrm{d})^{*}\right],
\end{aligned}
$$

with

$$
\begin{aligned}
& \mathrm{a}=(p, 0001|V| 0001) \doteq\left\langle{ }^{1} \mathrm{~S}_{0}|\hat{V}|{ }^{1} \mathrm{~S}_{0}\right\rangle, \\
& \mathrm{b}=i(p, 1101|V| 0001) \doteq\left\langle{ }^{3} \mathrm{P}_{0}|\hat{V}|{ }^{1} \mathrm{~S}_{0}\right\rangle, \\
& \mathrm{c}=(p, 0110|V| 0110) \doteq\left\langle{ }^{3} \mathrm{~S}_{1}|\hat{V}|{ }^{3} \mathrm{~S}_{1}\right\rangle, \\
& \mathrm{d}=-(p, 2110|V| 0110) \doteq\left\langle{ }^{3} \mathrm{D}_{1}|\hat{V}|^{3} \mathrm{~S}_{1}\right\rangle, \\
& \mathrm{e}=i(p, 1010|V| 0110) \doteq\left\langle{ }^{1} \mathrm{P}_{1}|\hat{V}|{ }^{3} \mathrm{~S}_{1}\right\rangle, \\
& \mathrm{f}=-i(p, 1111|V| 0111) \doteq\left\langle{ }^{3} \mathrm{P}_{1}|\hat{V}|{ }^{3} \mathrm{~S}_{1}\right\rangle,
\end{aligned}
$$

where the short notation

$$
\begin{aligned}
& (p, l S J T|V| 0 J J T) \\
& \quad \equiv\left(p, l L=0, \lambda=l, S J T|V| \mathrm{n}=1, \quad \mathrm{I}=\mathrm{L}=\lambda^{\prime}=0,\right. \\
& \left.S^{\prime}=J, J T\right)
\end{aligned}
$$

has been used for the matrix elements in Eq. (2.19).

Relationships between the matrix elements $\mathcal{M}(p l P L \lambda S J T ; \Lambda p)$, and the amplitudes a, b, c, d, e, and $f$ are shown in Appendix B. It can be seen that, although the derivation of the Eq. (3.6) for the $1 s_{1 / 2}$ orbital is mainly based on the relation

$$
\begin{aligned}
& \mathcal{M}(p l P L=0, \lambda=l S J T ; \Lambda p) \\
& \quad=(-)^{T+1}(P 0 \mid 10)(p, l S J ; T|V| 0 J J ; T),
\end{aligned}
$$

the one for the $1 p_{3 / 2}$ orbital is much more involved. In fact, in the latter case one has to consider all matrix elements $\mathcal{M}(p l P L=1, \lambda S J T ; \Lambda p)$ with $l+1 \geqslant \lambda \geqslant|l-1|$, each one of them containing the center-of-mass matrix element $(P 1 \mid 11)$ and one or two transition amplitudes $a, \cdots, f$.

When expressed in the framework of the OMEM, the SM matrix elements read

$$
\begin{aligned}
& \mathrm{a}=\frac{1}{\sqrt{2}}\left[C_{1}^{0}+C_{0}^{0}-3\left(S_{1}^{0}+S_{0}^{0}\right)\right] \\
& \mathrm{b}=-\frac{1}{\sqrt{2}}\left(P_{\pi}^{10}+P_{K_{1}}^{10}+P_{\eta}^{10}+P_{K_{0}}^{10}\right)+\sqrt{2}\left(\tilde{P}_{K_{1}^{*}}^{10}+\tilde{P}_{K_{0}^{*}}^{10}\right) \\
& \mathrm{c}=\frac{1}{\sqrt{2}}\left[S_{0}^{0}+C_{0}^{0}-3\left(S_{1}^{0}+C_{1}^{0}\right)\right] \\
& \mathrm{d}=2\left(3 T_{1}^{20}-T_{0}^{20}\right), \\
& \mathrm{e}=-\frac{1}{\sqrt{6}}\left[3\left(P_{\pi}^{10}+P_{K_{1}}^{10}+2 \tilde{P}_{K_{1}^{*}}^{10}\right)-P_{\eta}^{10}-P_{K_{0}}^{10}-2 \tilde{P}_{K_{0}^{*}}^{10}\right] \\
& \mathrm{f}=-\frac{1}{\sqrt{3}}\left[P_{\pi}^{10}-P_{K_{1}}^{10}+P_{\eta}^{10}-P_{K_{0}}^{10}\right] .
\end{aligned}
$$

The radial matrix elements $S, C, T, P, \tilde{P}$ are defined in Appendix A and are related to those defined Ref. [8], namely, $\mathrm{S}, \mathrm{C}, \mathrm{T}, \mathrm{P}, \tilde{\mathrm{P}}$, as $\mathrm{S}=S(P 0 \mid 10)$, etc. As indicated in the same appendix the subindices refer to isospin and the superindices to angular momentum transitions.

Although the SM leads to a NOSK-like expression for $a_{\Lambda}$ within the s-WA, both for ${ }_{\Lambda}^{5} \mathrm{He}$ and ${ }_{\Lambda}^{12} \mathrm{C}$, there are several differences between the SM matrix elements and those in the NOSK formula, and this is the reason for the symbol $\doteq$ in Eq. (3.7):

(i) The first ones depend on the relative momentum $p$, and the second ones do not.

(ii) $a, \cdots, f$ in Eq. (1.1) are in units of $\mathrm{MeV}^{-2}$, whereas $\mathrm{a}, \cdots, f$ in Eq. (3.2) are in units of $\mathrm{MeV}^{-1 / 2}$. This is due to the fact that the radial wave functions for the initial states are different.

(iii) As pointed out in Ref. [10], they differ as well by the phase factor $(-)^{S+J} i^{-l}$ that appears in Eq. (3.7), where the first correction is due to the change in ordering in the Clebsch-Gordan couplings for the spins, and the second one, to the fact that we do not include the phase $i^{l}$ in the final partial-wave radial function.

There are still two, at first glance, quite important differences between the NOSK formula [Eq. (1.1)] and our SM result. They come from the presence of the spherical harmonic and the integration in Eq. (3.5). Thus, to make them still more similar with each other, a few further approximations, which we feel are physically quite sound, are done:

(i) We assume that the $\epsilon$ 's do not play a significant role in Eq. (2.15). Thus, the liberated energy $\Delta_{\mathrm{j}_{p}}$ is approximated by $\Delta=M_{\Lambda}-M_{N}$, which means that in Eq. (3.5) is $d U_{s_{1 / 2}}=d U_{p_{3 / 2}}$.

(ii) The decay is basically back to back; therefore $\theta_{p} \cong 0$, and

$$
Y_{1,0}\left(\theta_{p}, 0\right) \cong Y_{1,0}(0,0)=\sqrt{3 / 4 \pi}
$$

(iii) The amplitudes $\mathcal{I}_{\kappa}(p ; 0)$ can be computed at $p \cong p_{\Delta}=$ $\sqrt{M_{N} \Delta}(P \cong 0)$ and factored out of the integrals. We 
end up with

$$
\omega_{\kappa}=\hat{\kappa} \mathcal{I}_{\kappa}\left(p=p_{\Delta} ; 0\right) \sum_{\mathrm{L}} \mathcal{J}_{\mathrm{L}},
$$

where

$$
\mathcal{J}_{\mathrm{L}}=\frac{2 M_{N}}{\pi} \int_{0}^{P_{\Delta}} P^{2} \sqrt{P_{\Delta}^{2}-P^{2}} \mathcal{O}(P ; \mathrm{L}) d P
$$

and $P_{\Delta}=2 \sqrt{M_{N} \Delta}=815 \mathrm{MeV}$. The essential point here is that, as shown in Eq. (2.19), the center-ofmass overlaps $\mathcal{O}(P ; L)$ have a Gaussian behavior in the variable $P$, and consequently the phase-space factors $P^{2} \sqrt{P_{\Delta}^{2}-P^{2}} \mathcal{O}(P ; \mathrm{L})$ in Eq. (3.13) are rather narrow peaks at $\sim 200 \mathrm{MeV}$. However, we have tested numerically that the amplitudes $\mathcal{I}_{\kappa}(p ; 0)$ have a very smooth dependence on $P$ in the range $0 \leqslant P \leqslant 300 \mathrm{MeV}$ $\left(p_{\Delta} \geqslant p \geqslant 380 \mathrm{MeV}\right)$.

Finally, the integrals $\sum_{L} \mathcal{J}_{L}$ cancel out in the numerator and the denominator in Eq. (2.17), and we obtain

$$
a_{\Lambda}=\sqrt{3} \frac{\mathcal{I}_{1}\left(p=p_{\Delta} ; 0\right)}{\mathcal{I}_{0}\left(p=p_{\Delta} ; 0\right)},
$$

which is the NOSK-like formula that we have been searching for.

We have also shown that

$$
\mathcal{J}_{0} \cong \mathcal{J}_{1} \cong 2 M_{N} p_{\Delta},
$$

which is consistent with the result [[8], Eq. (5.3)], and, together with Eq. (3.3), reveals that within the s-WA:

$$
\omega_{\kappa}\left(s_{1 / 2}\right) \cong \omega_{\kappa}\left(p_{3 / 2}\right) \text {. }
$$

That is, the $s_{1 / 2}$ and $p_{3 / 2}$ states contribute roughly by the same amounts, for both the proton-induced decay rate $\Gamma_{p} \equiv \omega_{0}$ and the numerator $\omega_{1}$ in the Eq. (2.17). It is worth noting that this is not valid in the case of the neutron-induced decay rate $\Gamma_{n}$, where, due to the Pauli principle, the $1 s_{1 / 2}$-state contribution is always larger [8] than that of the $1 p_{3 / 2}$ state.

For the sake of consistence, the proton-induced decay rate has to be evaluated from

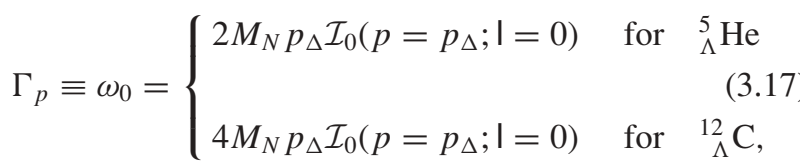

when the expression (3.14) is used for the asymmetry parameter. Note that the above result is a simple explanation for why

$$
\Gamma_{p}\left({ }_{\Lambda}^{12} \mathrm{C}\right) \cong 2 \Gamma_{\mathrm{p}}\left({ }_{\Lambda}^{5} \mathrm{He}\right) .
$$

We would like to stress that this is a purely kinematical result, and therefore it does not depend on the dynamics involved in the NMWD process.

As an application of the formalism developed here we exhibit the results for $a_{\Lambda}$ within the simple one-pion exchange model (OPEM) and within the $\pi+K$ model. Employing Eqs. (3.14), (A1), and (A2), we obtain, respectively:

$$
a_{\Lambda}^{\pi}=-\frac{2\left[\left(2 T_{\pi}^{20}-S_{\pi}^{0}\right) P_{\pi}^{10}\right]}{18\left(T_{\pi}^{20}\right)^{2}+\left(P_{\pi}^{10}\right)^{2}+3\left(S_{\pi}^{0}\right)^{2}}
$$

and

$$
a_{\Lambda}^{\pi+K}=-\frac{2\left[2\left(3 T_{1}^{20}-T_{K_{0}}^{20}\right) P_{\pi}^{10}-3\left(P_{\pi}^{10}-P_{K_{0}}^{10}\right) S_{1}^{0}-\left(2 P_{\pi}^{10}+3 P_{K_{1}}^{10}\right) S_{K_{0}}^{0}\right]}{6\left(3 T_{1}^{20}-T_{K_{0}}^{20}\right)^{2}+3\left(P_{\pi}^{10}\right)^{2}+2 P_{\pi}^{10}\left(2 P_{K_{1}}^{10}-P_{K_{0}}^{10}\right)+\left(P_{K_{0}}^{10}\right)^{2}+9\left(S_{1}^{0}\right)^{2}+3\left(P_{K_{1}}^{10}\right)^{2}+3\left(S_{K_{0}}^{0}\right)^{2}},
$$

where $T_{1}^{20}=T_{\pi}^{20}+T_{K_{1}}^{20}$ and $S_{1}^{0}=S_{\pi}^{0}+S_{K_{1}}^{0}$. We remark that gets: these results are valid for both ${ }_{\Lambda}^{5} \mathrm{He}$ and ${ }_{\Lambda}^{12} \mathrm{C}$.

$$
a_{\Lambda}^{\pi} \cong-\frac{4 T_{\pi}^{20} P_{\pi}^{10}}{18\left(T_{\pi}^{20}\right)^{2}+\left(P_{\pi}^{10}\right)^{2}}
$$

\section{NUMERICAL RESULTS}

In Tables I and II are compared the exact calculations for the asymmetry parameter $a_{\Lambda}^{\text {exact }}$, evaluated from Eqs. (2.17) and (2.18), with the approximated ones $a_{\Lambda}^{\text {approx }}$, obtained from Eq. (3.14). We see that the agreement between $a_{\Lambda}^{\text {exact }}$ and $a_{\Lambda}^{\text {approx }}$ is quite good for both ${ }_{\Lambda}^{5} \mathrm{He}$ and ${ }_{\Lambda}^{12} \mathrm{C}$. It can be seen from Table II that the relation (3.16) is fairly well fulfilled, which in turn implies the validity of Eq. (3.18).

Next, we briefly discuss the results within the OPEM and within the $\pi+K$ model for the individual matrix elements listed in Table III. It is seen from this table and Eq. (3.19) that the contribution of the scalar matrix element $S_{\pi}^{0}$ is small when compared with those coming from $T_{\pi}^{20}$ and $P_{\pi}^{10}$. Thus, one
TABLE I. Exact $\left(a_{\Lambda}^{\text {exact }}\right)$ and approximate $\left(a_{\Lambda}^{\text {approx }}\right)$ results for the asymmetry parameter $a_{\Lambda}$ in ${ }_{\Lambda}^{5} \mathrm{He}$. $a_{\Lambda}^{\text {exact }}$ is evaluated from Eqs. (2.17) and (2.18), and $a_{\Lambda}^{\text {approx }}$ from Eq. (3.14).

\begin{tabular}{lcc}
\hline \hline OMEM & $a_{\Lambda}^{\text {exact }}$ & $a_{\Lambda}^{\text {approx }}$ \\
\hline$\pi$ & -0.4354 & -0.4351 \\
$(\pi, \eta, K)$ & -0.5652 & -0.5852 \\
$\pi+\rho$ & -0.2449 & -0.2665 \\
$(\pi, \eta, K)+$ & -0.5117 & -0.5131 \\
$\left(\rho, \omega, K^{*}\right)$ & & \\
\hline \hline
\end{tabular}


TABLE II. Exact ( $\left.a_{\Lambda}^{\text {exact }}\right)$ and approximate $\left(a_{\Lambda}^{\text {approx }}\right)$ results for the asymmetry parameter $a_{\Lambda}$ in ${ }_{\Lambda}^{12} \mathrm{C} a_{\Lambda}^{\text {exact }}$ is evaluated from Eqs. (2.17) and (2.18), and $a_{\Lambda}^{\text {approx }}$ from Eq. (3.14). The HOC are given in Eqs. (A3) and (A4).

\begin{tabular}{lcccccc}
\hline \hline Approximation & $\omega_{0}\left(1 s_{1 / 2}\right)$ & $\omega_{0}\left(1 p_{3 / 2}\right)$ & $\omega_{1}\left(1 s_{1 / 2}\right)$ & $\omega_{1}\left(1 p_{3 / 2}\right)$ & $a_{\Lambda}^{\text {exact }}$ & $a_{\Lambda}^{\text {approx }}$ \\
\hline$\pi$ & & & & & & \\
With HOC & 0.4111 & 0.4724 & -0.1830 & -0.1990 & -0.4324 & \\
Without HOC & 0.4111 & 0.4327 & -0.1830 & -0.1863 & -0.4377 & -0.4501 \\
$(\pi, \eta, K)$ & & & & & & \\
With HOC & 0.2788 & 0.3161 & -0.1580 & -0.1707 & -0.5526 & \\
Without HOC & 0.2788 & 0.2811 & -0.1580 & -0.1569 & -0.5624 & -0.5860 \\
$\pi+\rho$ & & & & & & \\
With HOC & 0.4138 & 0.4607 & -0.0984 & -0.1096 & -0.2379 & \\
Without HO & 0.4138 & 0.4220 & -0.0984 & -0.1020 & -0.2398 & -0.2554 \\
$(\pi, \eta, K)+$ & & & & & & \\
$\left(\rho, \omega, K^{*}\right)$ & & & & & & \\
With HOC & 0.4391 & 0.4803 & -0.2300 & -0.2378 & -0.5088 & \\
Without HO & 0.4391 & 0.4477 & -0.2300 & -0.2271 & -0.5154 & -0.5306 \\
\hline \hline
\end{tabular}

which means that $a_{\Lambda}$ is large and negative in the OPEM, due to the interplay between the PC tensor $\left(T_{\pi}^{20}\right)$ and $P V$ dipole $\left(P_{\pi}^{10}\right)$ matrix elements.
In the same way from Table III one can easily see that the Eq. (3.20) can be approximated as:

$$
a_{\Lambda}^{\pi+K} \cong-\frac{2\left[2\left(3 T_{1}^{20}-T_{K_{0}}^{20}\right) P_{\pi}^{10}-3\left(P_{\pi}^{10}-P_{K_{0}}^{10}\right) S_{1}^{0}\right]}{6\left(3 T_{1}^{20}-T_{K_{0}}^{20}\right)^{2}+3\left(P_{\pi}^{10}\right)^{2}+2 P_{\pi}^{10}\left(2 P_{K_{1}}^{10}-P_{K_{0}}^{10}\right)+\left(P_{K_{0}}^{10}\right)^{2}+9\left(S_{1}^{0}\right)^{2}} .
$$

Thus the inclusion of the kaon modifies the above picture to a great extent. The matrix element $T_{\pi}^{20}$ goes now into the significantly smaller term $\left(T_{1}^{20}-T_{K_{0}}^{20} / 3\right)$, which would increase $a_{\Lambda}$ by the factor $T_{\pi}^{20} /\left(T_{1}^{20}-T_{K_{0}}^{20} / 3\right) \cong 1.5$. However, as can be seen from Table III, this effect is counterbalanced to a great extent by the large term $3\left(P_{\pi}^{10}\right)^{2}$ in the denominator, which now becomes more relevant in comparison with the tensor contribution. The kaon dipole and scalar contributions are also appreciable and we end up with a $a_{\Lambda}^{\pi+K}$ which is $\cong 25 \%$ larger than $a_{\Lambda}^{\pi}$. We note that, whereas the contribution of $S_{\pi}^{0}$ was neglected in $a_{\Lambda}^{\pi}$, that of $S_{1}^{0}$ is retained in $a_{\Lambda}^{\pi+K}$ because of the coherent contribution between $S_{\pi}^{0}$ and $S_{K_{1}}^{0}$.

By employing Eqs. (A1) and (A2) similar discussions can be performed for the exchanges of other mesons. In particular, one sees from Tables I and II that only the $\rho$ meson can diminish the value of the intrinsic $\Lambda$ asymmetry. From the last table it is not difficult to figure out that this comes from the destructive interference between the $\pi$ and $\rho$ mesons in the numerator of Eq. (2.17).

\section{SUMMARIZING CONCLUSIONS AND FINAL REMARKS}

In summary, by employing the s-WA and making use of a few plausible assumptions, we have succeeded in shaping the SM formalism for the asymmetry parameter $a_{\Lambda}$ into
NOSK-like formulas (3.5) and/or (3.14), which, in contrast to Eq. (1.1), are valid for finite hypernuclei. The new formalism (i) makes the theoretical evaluation of $a_{\Lambda}$ more transparent, (ii) explains clearly why the one-meson exchange model is unable to account for the experimental data of ${ }_{\Lambda}^{5} \mathrm{He}$, and (iii) helps to advance knowledge of the NMWD in general.

TABLE III. Nuclear matrix elements in units of $\mathrm{MeV}^{-1 / 2}$.

\begin{tabular}{lrr}
\hline \hline Matrix element & \multicolumn{1}{c}{${ }_{\Lambda}^{5} \mathrm{He}$} & \multicolumn{1}{c}{${ }_{\Lambda}^{12} \mathrm{C}$} \\
\hline$\pi$ & & \\
$T_{\pi}^{20}$ & -3.2402 & -3.7132 \\
$P_{\pi}^{10}$ & -8.0573 & -10.0379 \\
$S_{\pi}^{0}$ & 0.3876 & 0.4088 \\
$a_{\Lambda}^{\pi}$ & -0.4351 & -0.4501 \\
$K$ & & \\
$T_{K_{0}}^{20}$ & 0.4010 & 0.4313 \\
$T_{K_{1}}^{20}$ & 1.3438 & 1.4455 \\
$P_{K_{0}}^{10}$ & 4.8025 & 5.6107 \\
$P_{K_{1}}^{10}$ & 0.7388 & 0.8632 \\
$S_{K_{0}}^{0}$ & 0.1571 & 0.2238 \\
$S_{K_{1}}^{0}$ & 0.5265 & 0.7501 \\
$a_{\Lambda}^{\pi+K}$ & -0.5389 & -0.5500 \\
\hline \hline
\end{tabular}


It is still an open problem whether the result (2.18), and therefore the formulas (3.5), (3.14), (4.1), and (4.2), are of general validity. Their derivation is based on the properties of the single-proton spectroscopic amplitude between the core state $\left|J_{C}\right\rangle$ and the final states $\left|J_{F}\right\rangle$, which in the extreme SM, adopted here, have the simple expression [3, Eq. (31)]:

$$
\left\langle J_{C}|| a_{j_{p}}^{\dagger}|| J_{F}\right\rangle=(-)^{J_{F}+J_{C}+j_{p}} \hat{J}_{F} .
$$

This result allows us to perform the analytic summation on $J_{F}$, and $a_{\Lambda}$ becomes independent of the nuclear structure of the final states [cf. Eq. (2.17)]. However, because of the Pauli principle, it is only valid for hypernuclei with all single-particle proton subshells totally full, such as happens in ${ }_{\Lambda}^{5} \mathrm{He}$ and ${ }_{\Lambda}^{12} \mathrm{C}$. That is, we still do not know whether the Eq. (2.17), and all the developments presented here, can be used for other polarized hypernuclei, such as ${ }_{\Lambda}^{11} \mathrm{~B}$. Very likely it does, but this has to be proved!

Quite recently, and after the present work had been basically finished, the Barcelona group [15] has stated that a chirally motivated $2 \pi$-exchange mechanism of D. Jido, E. Oset, and J. E. Palomar [16] strongly affects the OMEM amplitudes a and $\mathrm{C}$ in the Eq. (3.10), producing in this way results that are consistent within the experimental data. Within the OMEM these two amplitudes are negative and of similar magnitudes due to the dominance of the central spin-isospin flipping matrix element $S_{1}^{0}$ in both of them. We feel that for a more thorough discussion of the interplay between the two transition mechanisms, it might be convenient to extend the formalism developed here by incorporating the $2 \pi$ exchanges into the Eqs. (3.10) and (3.20).

Last but not least, the very simple form of Eqs. (3.5) and (3.14) suggests that it might be possible to derive these expressions by more elementary considerations, instead of performing a very heavy Racah algebra, which has been done here. This would be highly desirable, but so far we have not been able to find such a simple argument.

\section{ACKNOWLEDGMENTS}

Two of us (C.B. and F.K.) are members of CONICET (Argentina). One of us (F.K.) acknowledges the support of FAPESP (São Paulo, Brazil). We are grateful to Gordana Tadić and Mahir S. Hussein for critical reading of the manuscript.

\section{APPENDIX A: FORMULAE FOR $\mathcal{I}_{\kappa}(p$; I) WITHIN THE OMEM}

The transition amplitudes [Eqs. (3.3) and (3.4)] that appear in Eq. (3.1) are:

$$
\begin{aligned}
\mathcal{I}_{0}(p & ; \mathrm{I}=0) \\
= & 2\left[6\left(3 T_{1}^{20}-T_{0}^{20}\right)^{2}+3\left(S_{0}^{0}\right)^{2}+9\left(S_{1}^{0}\right)^{2}+\left(C_{0}^{0}\right)^{2}\right. \\
& +7\left(C_{1}^{0}\right)^{2}-4 C_{0}^{0} C_{1}^{0}+12 C_{1}^{0} S_{1}^{0}-6 C_{0}^{0} S_{1}^{0}-6 C_{1}^{0} S_{0}^{0} \\
& +3\left(P_{\pi}^{10}\right)^{2}+\left(P_{\eta}^{10}\right)^{2}+3\left(P_{K_{1}}^{10}\right)^{2}+\left(P_{K_{0}}^{10}\right)^{2}+10\left(\tilde{P}_{K_{1}^{*}}^{10}\right)^{2} \\
& +2\left(\tilde{P}_{K_{0}^{*}}^{10}\right)^{2}-2 P_{\eta}^{10} P_{K_{1}}^{10}+2 P_{\pi}^{10}\left(2 P_{K_{1}}^{10}-P_{K_{0}}^{10}\right.
\end{aligned}
$$

$$
\begin{aligned}
& \left.+4 \tilde{P}_{K_{1}^{*}}^{10}-2 \tilde{P}_{K_{0}^{*}}^{10}\right)+4 P_{K_{1}}^{10}\left(2 \tilde{P}_{K_{1}^{*}}^{10}-\tilde{P}_{K_{0}^{*}}^{10}\right) \\
& \left.-4 \tilde{P}_{K_{1}^{*}}^{10}\left(P_{\eta}^{10}+P_{K_{0}}^{10}+\tilde{P}_{K_{0}^{*}}^{10}\right)\right],
\end{aligned}
$$

$$
\begin{aligned}
\mathcal{I}_{1}(p ; \mathrm{I} & =0) \\
& =\frac{4}{\sqrt{3}}\left[3\left(P_{\pi}^{10}-P_{K_{0}}^{10}+2 \tilde{P}_{K_{1}^{*}}^{10}\right) S_{1}^{0}+\left(2 P_{\pi}^{10}-P_{\eta}^{10}\right.\right. \\
& \left.+3 P_{K_{1}}^{10}+4 \tilde{P}_{K_{1}^{*}}^{10}-2 \tilde{P}_{K_{0}^{*}}^{10}\right) S_{0}^{0}+\left(P_{\eta}^{10}-3 P_{K_{1}}^{10}-2 P_{K_{0}}^{10}\right. \\
& \left.+2 \tilde{P}_{K_{0}^{*}}^{10}\right) C_{1}^{0}-\left(P_{\pi}^{10}-P_{K_{0}}^{10}+2 \tilde{P}_{K_{1}^{*}}^{10}\right) C_{0}^{0}-2\left(P_{\pi}^{10}\right. \\
& \left.\left.+P_{\eta}^{10}-\tilde{P}_{K_{1}^{*}}^{10}-\tilde{P}_{K_{0}^{*}}^{10}\right)\left(3 T_{1}^{20}-T_{0}^{20}\right)\right],
\end{aligned}
$$

$$
\begin{aligned}
\mathcal{I}_{0}(p ; \mid & =1) \\
& =6\left(S_{0}^{1}\right)^{2}+42\left(S_{1}^{1}\right)^{2}-24 S_{0}^{1} S_{1}^{1}+2\left(C_{0}^{1}\right)^{2}+6\left(C_{1}^{1}\right)^{2} \\
& -24 C_{1}^{1} S_{1}^{1}+12 C_{1}^{1} S_{0}^{1}+12 C_{0}^{1} S_{1}^{1}+\frac{6}{5}\left(T_{0}^{11}+T_{1}^{11}\right)^{2} \\
& +\frac{54}{5}\left(T_{0}^{31}+T_{1}^{31}\right)^{2}+14\left(P_{\pi}^{21}\right)^{2}+2\left(P_{\eta}^{21}\right)^{2}+8\left(P_{K_{1}}^{21}\right)^{2} \\
& +\frac{4}{3}\left(P_{K_{0}}^{21}\right)^{2}+14\left(\tilde{P}_{K_{1}^{*}}^{21}\right)^{2}+\frac{10}{3}\left(\tilde{P}_{K_{0}^{*}}^{21}\right)^{2}+4 P_{\eta}^{21} P_{K_{1}}^{21} \\
& -4 P_{\pi}^{21}\left(2 P_{\eta}^{21}+2 P_{K_{1}}^{21}-P_{K_{0}}^{21}+4 \tilde{P}_{K_{1}^{*}}^{21}-2 \tilde{P}_{K_{0}^{*}}^{21}\right) \\
& +4 P_{K_{1}}^{21}\left(-P_{K_{0}}^{21}-\tilde{P}_{K_{1}^{*}}^{21}+\tilde{P}_{K_{0}^{*}}^{21}\right)+4 \tilde{P}_{K_{1}^{*}}^{21}\left(2 P_{\eta}^{21}+P_{K_{0}}^{21}\right. \\
& \left.-\tilde{P}_{K_{0}^{*}}^{21}\right)+\frac{4}{3} P_{K_{0}}^{21} \tilde{P}_{K_{0}^{*}}^{21}+\frac{2}{3}\left(P_{K_{0}}^{01}\right)^{2}+6\left(P_{K_{1}}^{01}\right)^{2}+\frac{2}{3}\left(\tilde{P}_{K_{0}^{*}}^{01}\right)^{2} \\
& +6\left(\tilde{P}_{K_{1}^{*}}^{01}\right)^{2}-\frac{4}{3} P_{K_{1}}^{01} P_{K_{0}}^{01}-\frac{4}{3} \tilde{P}_{K_{0}^{*}}^{01}\left(P_{K_{0}}^{01}-3 P_{K_{1}}^{01}+3 \tilde{P}_{K_{1}^{*}}^{01}\right) \\
& +4 \tilde{P}_{K_{1}^{*}}^{01}\left(P_{K_{0}}^{01}-3 P_{K_{1}}^{01}\right),
\end{aligned}
$$

and

$$
\begin{aligned}
\mathcal{I}_{1}(p ; & \mathrm{I}=1) \\
& =\frac{4}{3 \sqrt{3}}\left\{\left(9 P_{\pi}^{21}+6 P_{\eta}^{21}+3 P_{K_{1}}^{21}+8 P_{K_{0}}^{21}+15 \tilde{P}_{K_{1}^{*}}^{21}\right.\right. \\
& \left.+13 \tilde{P}_{K_{0}^{*}}^{21}\right) S_{1}^{1}-\left(3 P_{\eta}^{21}+6 P_{K_{1}}^{21}+P_{K_{0}}^{21}+3 \tilde{P}_{K_{1}^{*}}^{21}\right. \\
& \left.+5 \tilde{P}_{K_{0}^{*}}^{21}\right) S_{0}^{1}+\frac{1}{2}\left(9 P_{\pi}^{21}-3 P_{\eta}^{21}-15 P_{K_{1}}^{21}+5 P_{K_{0}}^{21}\right. \\
& \left.+6 \tilde{P}_{K_{1}^{*}}^{21}-2 \tilde{P}_{K_{0}^{*}}^{21}\right)\left[\frac{1}{2}\left(C_{1}^{1}+C_{0}^{1}\right)+\frac{2}{5}\left(T_{1}^{11}+T_{0}^{11}\right)\right] \\
& -\frac{27}{5}\left(3 P_{\pi}^{21}-P_{\eta}^{21}-3 \tilde{P}_{K_{1}^{*}}^{21}+\tilde{P}_{K_{0}^{*}}^{21}\right)\left(T_{1}^{31}+T_{0}^{31}\right) \\
& +\left(3 P_{K_{1}}^{01}-P_{K_{0}}^{01}-3 \tilde{P}_{K_{1}^{*}}^{01}+\tilde{P}_{K_{0}^{*}}^{01}\right)\left(S_{1}^{1}+S_{0}^{1}+C_{1}^{1}+C_{0}^{1}\right. \\
& \left.\left.-T_{1}^{11}-T_{0}^{11}\right)\right\} .
\end{aligned}
$$

It should be noted that the formulas for $\mathcal{I}_{0}(p ;$ l) have been presented before in Ref. [8, Eq. (4.19)], and only the results for $\mathcal{I}_{1}(p ; \mid)$ are new. The radial matrix elements $S, C, T, P, \tilde{P}$ have the same meaning as the factors, S, C, T, P, $\tilde{\mathrm{P}}$ in Ref. [8] and are related to them as $\mathrm{S}=S(P 0 \mid 10)$, etc. Nevertheless, to facilitate the reading of the article we repeat their definitions also in the present work.

The parity conserving nuclear matrix elements are:

$$
\begin{aligned}
& C_{M}^{l}(p)=\mathcal{B}_{M}^{\prime}\left(p|| f_{M}|1|\right), \quad \text { for } \quad M=\pi, \eta, K, \rho, \omega, K^{*} \\
& S_{M}^{l}(p)=\mathcal{B}_{M}\left(p|| f_{M}^{S}|1|\right) \times\left\{\begin{array}{l}
1 \text { for } M=\pi, \eta, K \\
2 \text { for } M=\rho, \omega, K^{*}
\end{array}\right.
\end{aligned}
$$




$$
T_{M}^{l l}(p)=\mathcal{B}_{M}\left(p l\left|f_{M}^{T}\right| 1 \mid\right) \times\left\{\begin{array}{c}
1 \text { for } M=\pi, \eta, K \\
-1 \text { for } M=\rho, \omega, K^{*}
\end{array} .\right.
$$

and the parity-violating ones are:

$$
\begin{aligned}
P_{M}^{l l}(p) & =\mathcal{A}_{M}\left(p l\left|f_{M}^{(-)}\right| 1 \mid\right), \\
Q_{M}^{l l}(p) & =\mathcal{A}_{M}^{\prime}\left(p l\left|f_{M}^{(+)}\right| 1 \mid\right) .
\end{aligned}
$$

The radial form factors $\left(p|| f_{M}|1|\right),\left(p|| f_{M}^{S}|1|\right),\left(p l\left|f_{M}^{T}\right| 1 \mid\right)$, and $\left(p l\left|f_{M}^{( \pm)}\right| 1 \mathrm{l}\right)$, and the coupling constants $\mathcal{A}_{M}, \mathcal{A}_{M}^{\prime}, \mathcal{B}_{M}$, and $\mathcal{B}_{M}^{\prime}$ are given in Ref. [8].

The compact notations are also used:

$$
C_{0}=\frac{\tau=0}{C_{\omega}+C_{K_{0}}} \quad ; \quad C_{1}=C_{\rho}+C_{K_{1}^{*}},
$$

$S_{0}=S_{\eta}+S_{\omega}+S_{K_{0}}+S_{K_{0}^{*}} ; \quad S_{1}=S_{\pi}+S_{\rho}+S_{K_{1}}+S_{K_{1}^{*}}$,

$T_{0}=T_{\eta}+T_{\omega}+T_{K_{0}}+T_{K_{0}^{*}} ; \quad T_{1}=T_{\pi}+T_{\rho}+T_{K_{1}}+T_{K_{1}^{*}}$,

for the isoscalar $(\tau=0)$ and the isovector $(\tau=1)$ matrix elements, and

$$
\begin{array}{ccc}
\widetilde{P}_{\eta}=P_{\eta}-Q_{K_{0}^{*}}, & \widetilde{P}_{K_{0}}=P_{K_{0}}-Q_{\omega}, & \widetilde{P}_{K_{0}^{*}}=P_{K_{0}^{*}}+P_{\omega}, \\
\widetilde{P}_{\pi}=P_{\pi}-Q_{K_{1}^{*}}, & \widetilde{P}_{K_{1}}=P_{K_{1}}-Q_{\rho}, & \widetilde{P}_{K_{1}^{*}}=P_{K_{1}^{*}}+P_{\rho} .
\end{array}
$$

\section{APPENDIX B: RELATIONSHIP BETWEEN THE MATRIX ELEMENTS $\mathcal{M}(p l P L \lambda S J T ; \Lambda p)$, AND THE AMPLITUDES} $a, b, c, d, e$, AND $f$

$$
\begin{aligned}
\text { A. For } \mathcal{I}_{k}\left(\boldsymbol{p} ; \mathrm{j}_{p}=\mathbf{1} \boldsymbol{s}_{\mathbf{1} / \mathbf{2}}, \mathbf{0}\right): \\
\mathcal{M}(p 0, P 0,0001 ; \Lambda \mathrm{p})=\mathrm{a}(p)(P 0 \mid 10), \\
i \mathcal{M}(p 1, P 0,1101 ; \Lambda \mathrm{p})=\mathrm{b}(p)(P 0 \mid 10), \\
\mathcal{M}(p 0, P 0,0110 ; \Lambda \mathrm{p})=-\mathrm{c}(p)(P 0 \mid 10), \\
\mathcal{M}(p 2, P 0,2110 ; \Lambda \mathrm{p})=\mathrm{d}(p)(P 0 \mid 10), \\
i \mathcal{M}(p 1, P 0,1010 ; \Lambda \mathrm{p})=-\mathrm{e}(p)(P 0 \mid 10), \\
i \mathcal{M}(p 1, P 0,1111 ; \Lambda \mathrm{p})=-\mathrm{f}(p)(P 0 \mid 10) .
\end{aligned}
$$

B. For $\mathcal{I}_{\kappa}\left(p ; \mathrm{j}_{p}=1 p_{3 / 2}, 0\right)$ :

$$
\begin{aligned}
& \mathcal{M}(p 0, P 1,1011 ; \Lambda \mathrm{p})=\frac{1}{\sqrt{3}} \mathrm{a}(p)(P 1 \mid 11), \\
& \mathcal{M}(p 0, P 1,1110 ; \Lambda \mathrm{p})=-\frac{1}{\sqrt{6}} \mathrm{c}(p)(P 1 \mid 11), \\
& \mathcal{M}(p 0, P 1,1120 ; \Lambda \mathrm{p})=-\frac{1}{\sqrt{2}} \mathrm{c}(p)(P 1 \mid 11), \\
& i \mathcal{M}(p 1, P 1,0111 ; \Lambda \mathrm{p})=\frac{1}{3}\left(\frac{\mathrm{b}(p)}{\sqrt{3}}-\frac{\mathrm{f}(p)}{\sqrt{2}}\right)(P 1 \mid 11), \\
& i \mathcal{M}(p 1, P 1,1111 ; \Lambda \mathrm{p})=\left(\frac{\mathrm{b}(p)}{3}-\frac{\mathrm{f}(p)}{2 \sqrt{6}}\right)(P 1 \mid 11) \text {, } \\
& i \mathcal{M}(p 1, P 1,2111 ; \Lambda \mathrm{p})=\frac{\sqrt{5}}{3}\left(\frac{\mathrm{b}(p)}{\sqrt{3}}+\frac{\mathrm{f}(p)}{2 \sqrt{2}}\right)(P 1 \mid 11), \\
& i \mathcal{M}(p 1, P 1,1010 ; \Lambda \mathrm{p})=-\frac{1}{\sqrt{6}} \mathrm{e}(p)(P 1 \mid 11), \\
& i \mathcal{M}(p 1, P 1,2020 ; \Lambda \mathrm{p})=-\frac{1}{\sqrt{2}} \mathrm{e}(p)(P 1 \mid 11), \\
& i \mathcal{M}(p 1, P 1,1121 ; \Lambda \mathrm{p})=-\frac{1}{2 \sqrt{2}} \mathrm{f}(p)(P 1 \mid 11), \\
& i \mathcal{M}(p 1, P 1,2121 ; \Lambda \mathrm{p})=-\frac{\sqrt{3}}{2 \sqrt{2}} \mathrm{f}(p)(P 1 \mid 11), \\
& \mathcal{M}(p 2, P 1,1110 ; \Lambda \mathrm{p})=\frac{1}{2 \sqrt{2}} \mathrm{~d}(p)(P 1 \mid 11), \\
& \mathcal{M}(p 2, P 1,2110 ; \Lambda \mathrm{p})=\frac{1}{2 \sqrt{2}} \mathrm{~d}(p)(P 1 \mid 11), \\
& \mathcal{M}(p 2, P 1,1120 ; \Lambda \mathrm{p})=\frac{1}{10 \sqrt{2}} \mathrm{~d}(p)(P 1 \mid 11), \\
& \mathcal{M}(p 2, P 1,2120 ; \Lambda \mathrm{p})=\frac{\sqrt{21}}{5 \sqrt{2}} \mathrm{~d}(p)(P 1 \mid 11), \\
& \mathcal{M}(p 2, P 1,3120 ; \Lambda \mathrm{p})=\frac{\sqrt{21}}{5 \sqrt{2}} \mathrm{~d}(p)(P 1 \mid 11) .
\end{aligned}
$$

[1] W. M. Alberico and G. Garbarino, Phys. Rep. 369, 1 (2002); W. M. Alberico, G. Garbarino, A. Parreño, and A. Ramos, Phys. Rev. Lett. 94, 082501 (2005).

[2] S. Ajimura et al., Phys. Lett. B282, 293 (1992); S. Ajimura et al., Phys. Rev. Lett. 84, 4052 (2000); T. Maruta et al., Nucl. Phys. A754, 168c (2005).

[3] C. Barbero, A. P. Galeão, and F. Krmpotić, Phys. Rev. C 72, 035210 (2005).

[4] H. Nabetani, T. Ogaito, T. Sato, and T. Kishimoto, Phys. Rev. C 60, 017001 (1999).

[5] J. F. Dubach, G. B. Feldman, B. R. Holstein, and L. de la Torre, Ann. Phys. (N.Y.) 249, 146 (1996).

[6] M. B. Block and R. H. Dalitz, Phys. Rev. Lett. 11, 96 (1963).

[7] K. Sasaki, M. Izaki, and M. Oka, Phys. Rev. C 71, 035502 (2005).
[8] C. Barbero, D. Horvat, F. Krmpotić, T. T. S. Kuo, Z. Narančić, and D. Tadić, Phys. Rev. C 66, 055209 (2002).

[9] F. Krmpotić and D. Tadić, Braz. J. Phys. 33, 187 (2003).

[10] C. Barbero, C. De Conti, A. P. Galeão, and F. Krmpotić, Nucl. Phys. A726, 267 (2003).

[11] N. Austern, Direct Nuclear Reaction Theories (WileyInterscience, New York, 1970).

[12] A. Ramos, E. van Meijgaard, C. Bennhold, and B. K. Jennings, Nucl. Phys. A544, 703 (1992).

[13] M. Moshinsky, Nucl. Phys. 13, 104 (1959).

[14] K. Itonaga, T. Ueda, and T. Motoba, Phys. Rev. C 65, 034617 (2002).

[15] C. Chumillas, G. Garbarino, A. Parreño, and A. Ramos, arXiv: 0705.0231 [nucl-th].

[16] D. Jido, E. Oset, and J. E. Palomar, Nucl. Phys. A694, 525 (2001). 